\title{
EXPERIMENTAL EVALUATION OF A CASCADE CONTROL TECHNIQUE WITH FRICTION COMPENSATION FOR A FLEXIBLE JOINT ROBOT
}

\author{
A. R. G. Ramires* \\ garcia@gmail.com \\ R. Guenther ${ }^{\ddagger}$ \\ In Memoriam \\ E.R. De Pieri ${ }^{\dagger}$ \\ edsonedas.ufsc.br \\ J. F. Golin ${ }^{\ddagger}$ \\ golinedas.ufsc.br \\ *University of Vale de Itajaí, Computation Engineering, Campus São José \\ Rodovia SC 407, Km 4 88122-000 São José-SC, Brazil \\ ${ }^{\dagger}$ Department of Automation and Systems, Federal University of Santa Catarina \\ 88040-900 Florianópolis, SC, Brazil \\ ${ }^{\ddagger}$ Department of Mechanical Engineering, Federal University of Santa Catarina \\ 88040-900 Florianópolis, SC, Brazil
}

\begin{abstract}
This paper focuses on the experimental results of the position tracking control of a flexible joint robot manipulator. The applied control strategy is based on a well know cascade control methodology that divides the robot model into two subsystems: a link and a rotor subsystem. Thanks to this division a friction compensation based on the LuGre model is performed to the mechanical system. The main goal of the present work is to apply a systematic procedure to identify the friction model parameters and to analyse the control strategy performance when implemented to a robot prototype specially built for practical tests.
\end{abstract}

KEYWORDS: Robot control, joint flexibility, friction observer, friction compensation, cascade control.

\section{RESUMO}

Controle em Cascata com Compensação de Atrito para Robôs com Juntas Flexíveis: Teoria e Resultados Experi-

Artigo submetido em 20/12/2007 (Id.: 00841)

Revisado em 01/04/2008, 15/09/2008, 03/12/2008, 18/04/2009

Aceito sob recomendação da Editora Associada Profa. Anna Helena Reali Costa

\section{mentais}

Neste trabalho são apresentados resultados experimentais de seguimento de trajetória de posição de um robô manipulador com flexibilidade nas juntas. A estratégia de controle é baseada na metodologia do controle em cascata, dividindo o modelo do robô em dois subsistemas: o subsistema dos elos e o subsistema do rotor. Essa abordagem facilita a compensação do problema de atrito do subsistema mecânico ou dos elos. Usou-se para a compensação de atrito o modelo de LuGre. O principal objetivo da abordagem apresentada é fornecer um procedimento sistemático para identificação dos parâmetros do modelo de atrito e a análise de desempenho da estratégia proposta quando aplicada a um protótipo especialmente construído para testes experimentais.

PALAVRAS-CHAVE: Controle de Robôs, Robôs Flexíveis, Observador de Atrito, Controle em Cascata

\section{INTRODUCTION}

Undesirable elastic deformations may appear in fast robots equipped with gear-boxes, harmonic drives, chains or belts. The elastic deformation due to joint flexibility might cause positioning errors, increase the settling time and even produce torsional resonance and instability (Spong, 1987). Also, 
the elastic coupling introduces an additional degree of freedom at each joint. In particular, a robot with flexible transmissions has the double of degrees of freedom with respect to its rigid equivalent. Thus, this robot is a partially actuated system, since it has $2 n$ variables to be controlled through only $n$ control torques. Therefore, the usual methods for tracking control, such as the inverse dynamics formulation and the control based on Lyapunov, cannot be directly applied to that system (Readman, 1994).

Different control strategies for elastic joint robots have been developed in the literature. Among those strategies, one may cite PD control (Tomei, 1991; Dupont, 1994), dynamic feedback linearization methods, singular perturbation theory, robust and adaptive schemes (Nicosia and Tomei, 1988; Tomei, 2000), integrator backstepping based methodologies (Nicosia and Tomei, 1992) and cascade methodologies (Guenther and Hsu, 1993).

There are some important differences between those techniques. A basic comparative study can be found in (Brogliato et al., 1995). PD controllers, which work well for industrial robots, add some damping for the joint elasticity but their performance and robustness are very limited. The controller bandwidth has to be reduced until robustness against highly nonlinear dynamics is reached. Stability proofs for such controllers are more complicated than for the controllers using extensive model information. The singular perturbation is a simple two stage method, referred as corrective control, but it only works well in the case of weak flexibility. Adaptive versions of that control technique have been developed with good results (Ghorbel and Spong, 2000). An extension to consider the problem of position and force control of uncertain constrained flexible joint robots was presented in (Huang et al., 2006). The dynamic feedback linearization methods are quite simple to be used but in the most general cases the dynamics of flexible joint robots are not feedback linearizable. Also, the main disadvantage of feedback linearization is the necessity of the exact knowledge of the robot parameters and the need of joint acceleration and jerk measurements. To overcome these difficulties, adaptive and passivity based control techniques have been proposed (Fantoni and Lozano, 2000; Albu-Schäffer and Hirzinger, 2001). The backstepping schemes use the cascade decomposition property of the robot model (Kokotovic and Sussmann, 1989). The backstepping is a systematic procedure to control nonlinear systems but the designer should pay attention to overparametrization and time consuming computations. These latter are more involved than compared to the traditional cascade control procedure, for example. Also, in some algorithms, the measurement of joint accelerations is necessary. Using the cascade control methodology, a robust tracking of a reference model, avoiding overparametrization, was achieved (Wang and Khorrami, 2000). The cascade control schemes also use the system decompositions in small subsystems. The overall system is partitioned in such a way that the states of the first subsystem are the control variables to the following subsystem. In this technique, the model uncertainties can be taken into account by using an adaptive control scheme. With this methodology, the problem of measuring joint accelerations can be circumvented.

In robots with joint flexibilities, some important phenomena like actuator dynamics and friction are not accurately included in the design of control algorithms (Lozano et al., 1997; Ramirez et al., 2003). In fact, friction occurs in all mechanical systems,e.g. bearings, transmissions, hydraulic and pneumatic cylinders, valves, brakes and wheels (Canudas De Wit et al., 1995; Canudas De Wit and Lischinsky, 1997; Guenther et al., 2006; Lischinsky et al., 1999; Guenther and Perondi, 2004). In electrical driven robots the friction is always present in transmission mechanisms, rotor and joints (Canudas De Wit et al., 1995). Friction can lead to steady state or trajectory tracking errors (Dupont, 1994; Guenther and Perondi, 2002; Ramirez et al., 2002) or can lead to cycle limites in flexible robots as shown in (Jeon and Tomizuka, 2005). Friction compensation is desired to circumvent these problem and it is particularly difficult to be performed using a non-model based compensation (Lozano et al., 1997; Tomei, 2000) or even using a model-based compensation (Armstrong-Hélouvry, 1993; Canudas De Wit and Lischinsky, 1997) or using adaptive techniques (Xie, 2007).

Several models have been used to describe the friction phenomenon dynamics. In (Gomes and Santos da Rosa, 2003) a model is proposed to compensate friction effects. In a different structure (Canudas De Wit et al., 1995; C. Canudas de Wit, 2007) develop a friction estimation scheme based in the LuGre model to build a model-based fiction compensation. In (Gandhi et al., 2002) an extension of the LuGre model is proposed to compensate friction in harmonic drives. $\mathrm{Re}$ cently, a Generalized Maxwell-Slip model has been proposed by (Al-Bender et al., 2005) to describe the friction dynamics.

This paper proposes an experimental setup to analyse a cascade control methodology in which the mechanical system is interpreted as an interconnected system: a link subsystem driven by the elastic torque generated by the rotor subsystem. The cascade control strategy allows to introduce friction compensation in the mechanical system. For this purpose, a model-based friction compensation is adopted. It uses a friction estimation scheme based on the LuGre model (Canudas De Wit et al., 1995). The estimated friction torque is added to the reference torque generated by the position controller. This compensation assumes that the actuator has a fast and accurate torque response. The convergence of the tracking errors is demonstrated using the Lyapunov direct method, for the case where all the system parameters are known and there 
are not external forces. Experimental results illustrate the main properties of the proposed controller with the friction compensation model.

This paper is organized as follows. In Section 2, the model of the flexible joint robot manipulator with friction is recalled. The cascade control with friction compensation is proposed in Section 3. In Section 4, the stability of the proposed controller is demonstrated. Section 5 describes the off-line procedure for the identification of friction parameters. In Section 6 , the experiment is presented.

\section{MODEL OF RIGID AND FLEXIBLE ROBOTS WITH $N$ LINKS}

A mathematical model of a robot with $n$ rigid links connected by $n$ rotational or translation rigid joints is given by (Canudas De Wit et al., 1996)

$$
M\left(q_{1}\right) \ddot{q}_{1}+C\left(q_{1}, \dot{q}_{1}\right) \dot{q}_{1}+G\left(q_{1}\right)=\tau
$$

where the vectors $q_{1}, \dot{q}_{1}$ and $\ddot{q}_{1}$ represent, respectively, the link position, velocity and acceleration, $M\left(q_{1}\right)$ is the inertia matrix, $C\left(\dot{q}_{1}, q_{1}\right)$ represents the centrifuges and Coriolis terms, $G\left(q_{1}\right)$ represents the gravitational torques and $\tau$ is the control input. As pointed out in (Canudas De Wit et al., 1996), the elastic effects are small and can be neglected, the inertia matrix $M\left(q_{1}\right)$ is symmetric and positive definite and both $M\left(q_{1}\right)$ and $M^{-1}\left(q_{1}\right)$ are uniformly bounded for all $q_{1} \in \Re^{n}$, and a convenient choice of $C\left(\dot{q}_{1}, q_{1}\right)$ leads to an skew-symmetric matrix $\dot{M}-2 C$. This system is considered completely actuated in the sense that one torque is provided for each degree of freedom.

In order to obtain the mathematical model of a flexible joint robot, the links are supposed to be rigid, the joint flexibility is modeled as a torsional spring for rotational joints and as a linear spring for prismatic joints, and the inertia matrix takes into account the individual inertia of the links and rotors (Spong and Vidyasagar, 1989). Then, the dynamical model of a flexible joint robot can be written as

$$
\bar{M}\left(q_{1}\right) \ddot{q}+\bar{C}(\dot{q}, q) \dot{q}+\bar{G}\left(q_{1}\right)+\bar{K} q=\bar{\tau}
$$

where $q_{1}$ is associated to the position on the link, $q_{2}$ is associated to the position on the rotor and $q^{\mathrm{T}}=\left[\begin{array}{ll}q_{1}^{\mathrm{T}} & q_{2}^{\mathrm{T}}\end{array}\right]$. The elements $\bar{M}\left(q_{1}\right), \bar{C}(\dot{q}, q), \bar{G}\left(q_{1}\right), \bar{K}$ and $\bar{\tau}$ are described in (Canudas De Wit et al., 1996).

With the hypothesis introduced by (Tomei, 1991), (Canudas De Wit et al., 1996) and (Spong, 1987), it is possible to obtain a simplified model, given by

$$
\begin{aligned}
M\left(q_{1}\right) \ddot{q}_{1}+C\left(q_{1}, \dot{q}_{1}\right) \dot{q}_{1}+G\left(q_{1}\right) & =K\left(q_{2}-q_{1}\right) \\
J \ddot{q}_{2}+K\left(q_{2}-q_{1}\right) & =\tau
\end{aligned}
$$

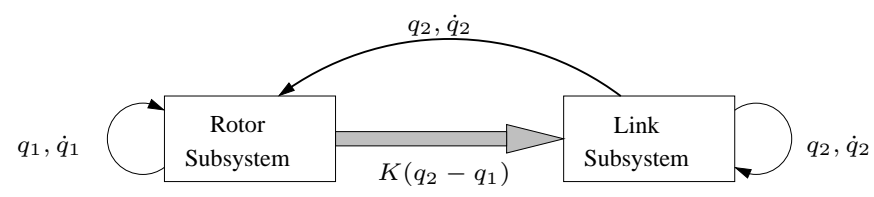

Figure 1: Cascade representation of flexible joint robots.

with $J=\operatorname{diag}\left(J_{1}, J_{2}, \ldots, J_{n}\right), J_{j}$ being the inertia associated to the rotor $j$, and $K=\operatorname{diag}\left(K_{1}, K_{2}, \ldots, K_{n}\right), K_{j}$ being the spring coefficient associated to joint $j$. Some robot configurations can be adequately modelled by (3), like manipulators with one rotational joint and one link or manipulators with two rotational joints and two links with perpendicular axes. In general, when the transmission reductions are important (for example when harmonic drivers are used) the simplified model (3) is valid.

The mathematical model (3) can be interpreted as two coupled subsystems: the first one represents the rigid robot model and the second one corresponds to the flexible joint dynamics. The coupling between the two models is given by the elastic torque represented by the term $K\left(q_{2}-q_{1}\right)$. The cascade interconnection of the two subsystem is shown in Fig. (1).

From the control point of view, the simplified model (3) describes a non negligible elastic displacement between each link and its corresponding rotor. On the other hand, the whole system becomes partially actuated, since for $2 n$ equations describing the dynamical behavior of the system there are only $n$ torque inputs. The main consequence of this behavior is to limit the application of classical controllers (as P, PD and PID) normally used to control rigid manipulators. Furthermore, for an effective control of flexible joint robots it is necessary to use more elaborated control strategies (possibly with friction compensation) and to take into account some robustness requirements.

\subsection{Friction Model}

In this paper, the LuGre model (Canudas De Wit et al., 1995) is used to represent the friction. This model can describe complex friction behavior, such as stick-slip motion, presliding displacement, Dahl and Stribeck effects and frictional lag.

The friction force is given by

$$
F=\Gamma_{0} z+\Gamma_{1} \dot{z}+\Gamma_{2} \dot{q}_{1}
$$

where $z$ is an internal state that describes the average elastic deflection of the contact surfaces during the stiction phases, $\Gamma_{0}$ is a diagonal matrix of the stiffness coefficient of the microscopic deformations $z$ during the pre-sliding displace- 
ments, $\Gamma_{1}$ is a diagonal matrix of the damping coefficients associated with $\dot{z}$ and $\Gamma_{2}$ is a diagonal matrix that represents the viscous friction.

The dynamics of the internal state $z$ is expressed by

$$
\dot{z}=\dot{q}_{1}-\Gamma_{0} \Psi\left(\dot{q}_{1}\right) z
$$

with

$$
\Psi\left(\dot{q}_{1}\right)=\operatorname{diag}\left(\frac{\left|\dot{q}_{11}\right|}{g\left(\dot{q}_{11}\right)}, \ldots, \frac{\left|\dot{q}_{1 n}\right|}{g\left(\dot{q}_{1 n}\right)}\right)
$$

where $g\left(\dot{q}_{1 i}\right)$ for $i=1, \ldots, n$ is a positive function that describes part of the steady state characteristics of the model for constant velocity motions. This function is given by

$$
g\left(\dot{q}_{1 i}\right)=F_{c i}+\left(F_{s i}-F_{c i}\right) e^{-\left(\frac{\dot{q}_{1 i}}{v_{s i}}\right)^{2}}
$$

where $F_{c i}$ is the Coulomb friction, $F_{s i}$ is the static friction and $v_{s i}$ is the Stribeck velocity and as stated in (Canudas De Wit et al., 1995), each function $g\left(\dot{q}_{1 i}\right)$ is upper bounded, i.e., $0<g\left(\dot{q}_{1 i}\right) \leq a_{i}$, for $i=1, \ldots, n$.

The simplified model (3) is modified to take into account the friction. Then,

$$
\begin{aligned}
M\left(q_{1}\right) \ddot{q}_{1}+C\left(q_{1}, \dot{q}_{1}\right) \dot{q}_{1}+G\left(q_{1}\right)+F & =K\left(q_{2}-q_{1}\right) \\
J \ddot{q}_{2}+K\left(q_{2}-q_{1}\right) & =\tau
\end{aligned}
$$

\section{CASCADE CONTROL WITH FRICTION COMPENSATION}

In this section, the cascade control with friction compensation is proposed for systems that may be represented by the model (8). Initially, the following errors are defined

$$
\begin{aligned}
\tilde{q}_{1} & =q_{1}-q_{1 d} \\
\tilde{q}_{2} & =q_{2}-q_{2 d} \\
\dot{q}_{r 1} & =\dot{q}_{1 d}-\Lambda_{1} \tilde{q}_{1} \\
s_{1} & =\dot{q}_{1}-\dot{q}_{r 1} \\
s_{2} & =\dot{\tilde{q}}_{2}+\Lambda_{2} \tilde{q}_{2}
\end{aligned}
$$

where $\Lambda_{1}$ and $\Lambda_{2}$ are diagonal positive definite matrices of gains, $q_{1 d}$ is the desired link position, $q_{2 d}$ is the desired rotor position and $\dot{q}_{r 1}$ is a reference for the link velocity.

The system (8) can be transformed into two cascade subsystems with inputs $v$ and $q_{2}$ using a suitable change of coordinates given by

$$
\begin{gathered}
\tau=J v+K\left(q_{2}-q_{1}\right) \\
q_{2 d}=K^{-1} u_{d}+q_{1}
\end{gathered}
$$

where $v$ is the control applied to the rotor subsystem and $u_{d}$ is the control for the link subsystem.

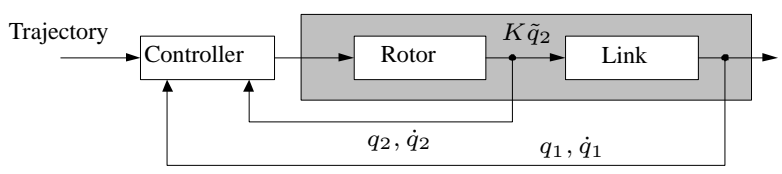

Figure 2: Cascade model.

Substituting (10) and (11) in (8), the system is rewritten as

$$
\begin{aligned}
M\left(q_{1}\right) \ddot{q}_{1}+C\left(q_{1}, \dot{q}_{1}\right) \dot{q}_{1}+G\left(q_{1}\right)+F & =u_{d}+K \tilde{q}_{2} \\
\ddot{q}_{2} & =v
\end{aligned}
$$

The robot system is then interpreted as an interconnected system like the one presented in Fig. 2.

The control project is performed through two steps: link control and rotor control. In addition, a friction observer is designed.

\subsection{Tracking Control of the Link Subsys- tem}

The control $u_{d}$ is designed using the property of passivity in robot manipulators(Canudas De Wit et al., 1996). Thus, $u_{d}$ is given by

$$
u_{d}=M\left(q_{1}\right) \ddot{q}_{r 1}+C\left(q_{1}, \dot{q}_{1}\right) \dot{q}_{r 1}+G\left(q_{1}\right)-K_{D 1} s_{1}+\hat{F}
$$

where $K_{D 1}$ is a diagonal positive definite matrix of gains and $\hat{F}$ is the estimated friction torque.

The closed loop form of this subsystem is obtained substituting (13) in the first equation of (12)

$$
M\left(q_{1}\right) \dot{s}_{1}+\left(C\left(q_{1}, \dot{q}_{1}\right)+K_{D 1}\right) s_{1}+F-\hat{F}=K \tilde{q}_{2}
$$

\subsection{Tracking Control of the Rotor Subsys- tem}

The control $v$ is chosen as

$$
v=\ddot{q}_{2 d}-\Lambda_{2} \dot{\tilde{q}}_{2}-J^{-1} K_{D 2} s_{2}
$$

where $K_{D 2}$ is a diagonal positive definite matrix of gains. Then, the control torque $\tau$ is given by

$$
\tau=J\left(\ddot{q}_{2 d}-\Lambda_{2} \dot{\tilde{q}}_{2}\right)-K_{D 2} s_{2}+K\left(q_{2}-q_{1}\right)
$$

The closed loop form of this subsystem is obtained substituting (15) in the second equation of (12)

$$
J \dot{s}_{2}+K_{D 2} s_{2}=0
$$




\subsection{Friction Observer}

According to (Canudas De Wit et al., 1995) the estimated friction $\hat{F}$ is

$$
\hat{F}=\Gamma_{0} \hat{z}+\Gamma_{1} \dot{\hat{z}}+\Gamma_{2} \dot{q}_{1}
$$

where $\hat{z}$ is the estimated internal state.

In (Guenther and Perondi, 2002), the following expression is proposed to model the dynamic of $\hat{z}$,

$$
\dot{\hat{z}}=\dot{q}_{1}-\Gamma_{0} \Psi\left(\dot{q}_{1}\right) \hat{z}-K_{e} \Gamma_{0} s_{1}
$$

where $K_{e}$ is diagonal positive definite matrix of gains.

The observer in (19) requires a slight modification to be used within the cascade control scheme. This modification is necessary because the derivative of the estimated friction is used to calculate the control signal in the cascade control strategy. Therefore, the function $\left|\dot{q}_{1 i}\right|$ has to be smoothed by a function $m\left(\dot{q}_{1 i}\right)$, like

$$
m\left(\dot{q}_{1 i}\right)=\frac{2}{\pi} \dot{q}_{1 i} \arctan \left(k_{v i} \dot{q}_{1 i}\right)
$$

where $k_{v i}$ is a positive constant (Guenther and Perondi, 2002) taken as $k_{v i}=2.0$ in the applications. This gives a new matrix $\hat{\Psi}\left(\dot{q}_{1}\right)$, defined as

$$
\hat{\Psi}\left(\dot{q}_{1}\right)=\operatorname{diag}\left(\frac{m\left(\dot{q}_{11}\right)}{g\left(\dot{q}_{11}\right)}, \ldots, \frac{m\left(\dot{q}_{1 n}\right)}{g\left(\dot{q}_{1 n}\right)}\right)
$$

and a new model for the dynamic of $\hat{z}$, which is used in this work,

$$
\dot{\hat{z}}=\dot{q}_{1}-\Gamma_{0} \hat{\Psi}\left(\dot{q}_{1}\right) \hat{z}-K_{e} \Gamma_{0} s_{1}
$$

Thus, the difference between the friction internal state $z$ and the estimated state $\hat{z}$ denoted by $\tilde{z}=z-\hat{z}$, has the time derivative

$$
\dot{\tilde{z}}=-\Gamma_{0} \hat{\Psi}\left(\dot{q}_{1}\right) \tilde{z}-\Gamma_{0} \Delta_{\Psi}\left(\dot{q}_{1}\right) z+K_{e} \Gamma_{0} s_{1}
$$

where $\Delta_{\Psi}\left(\dot{q}_{1}\right)$, defined as $\Delta_{\Psi}\left(\dot{q}_{1}\right)=\Psi\left(\dot{q}_{1}\right)-\hat{\Psi}\left(\dot{q}_{1}\right)$, is a residual difference caused by the use of $m\left(\dot{q}_{1 i}\right)$ instead of $\left|\dot{q}_{1 i}\right|$.

\section{STABILITY ANALYSIS}

The closed loop is constituted by the robot and the cascade controller, considering the friction observer. In the ideal case, in which all the system parameters are known, the convergence properties of the tracking errors are stated below.

Consider the nonnegative energy function

$$
V=V_{1}+V_{2}
$$

where $V_{1}$ and $V_{2}$ are nonnegative functions related to the link and the rotor subsystems, respectively, given by

$$
\begin{gathered}
V_{1}=\frac{1}{2} s_{1}^{\mathrm{T}} M\left(q_{1}\right) s_{1}+\frac{1}{2} \tilde{z}^{\mathrm{T}} K_{e}^{-1} \tilde{z}+\frac{1}{2} \tilde{q}_{1}^{\mathrm{T}} P_{1} \tilde{q}_{1} \\
V_{2}=\frac{1}{2} s_{2}^{\mathrm{T}} J s_{2}+\frac{1}{2} \tilde{q}_{2}^{\mathrm{T}} P_{2} \tilde{q}_{2}
\end{gathered}
$$

with $P_{1}=2 \Lambda_{1} K_{D 1}$ and $P_{2}=2 \Lambda_{2} K_{D 2}$.

The time derivative of $V_{1}$, substituting $M\left(q_{1}\right) \dot{s}_{1}$ by the expression obtained from (14) and $\dot{\tilde{z}}$ by the expression in (23) and using the property of skew symmetry of $\left(\frac{1}{2} \dot{M}\left(q_{1}\right)-C\left(q_{1}, \dot{q}_{1}\right)\right)$, is given by

$$
\begin{aligned}
& \dot{V}_{1}=-s_{1}^{\mathrm{T}}\left(K_{D 1}+\Gamma_{1} K_{e} \Gamma_{0}\right) s_{1}+s_{1}^{\mathrm{T}} \Gamma_{1} \Gamma_{0} \hat{\Psi}\left(\dot{q}_{1}\right) \tilde{z}+ \\
& \quad s_{1}^{\mathrm{T}} \Gamma_{1} \Gamma_{0} \Delta_{\Psi}\left(\dot{q}_{1}\right) z+s_{1}^{\mathrm{T}} K \tilde{q}_{2}-\tilde{z}^{\mathrm{T}} K_{e}^{-1} \Gamma_{0} \hat{\Psi}\left(\dot{q}_{1}\right) \tilde{z} \\
& \quad-\tilde{z}^{\mathrm{T}} K_{e}^{-1} \Gamma_{0} \Delta_{\Psi}\left(\dot{q}_{1}\right) z+\tilde{q}_{1}^{\mathrm{T}} P_{1} \dot{\tilde{q}}_{1}
\end{aligned}
$$

The time derivative of $V_{2}$, substituting $J \dot{s}_{2}$ by the expression obtained from (17), is given by

$$
\dot{V}_{2}=-s_{2}^{\mathrm{T}} K_{D 2} s_{2}+\tilde{q}_{2}^{\mathrm{T}} P_{2} \dot{\tilde{q}}_{2}
$$

Substituting $P_{1}, P_{2}, s_{1}$ and $s_{2}$ in equations (27) and (28), the time derivative of $V$ may be written as ${ }^{1}$

$$
\dot{V}=-\rho^{\mathrm{T}} N_{2} \rho+\rho^{\mathrm{T}} D
$$

with

$$
\begin{aligned}
& \rho^{\mathrm{T}}=\left[\begin{array}{lllll}
q_{1}^{\mathrm{T}} & \dot{q}_{1}^{\mathrm{T}} & q_{2}^{\mathrm{T}} & \dot{q}_{2}^{\mathrm{T}} & \eta^{\mathrm{T}}
\end{array}\right] \quad ; \quad \eta=\hat{\Psi}\left(\dot{q}_{1}\right) \tilde{z} \\
& N_{2}=\left[\begin{array}{ll}
N_{2_{11}} & N_{2_{12}} \\
N_{2_{21}} & N_{2_{22}}
\end{array}\right] \\
& N_{2_{11}}=\left[\begin{array}{cc}
\Lambda_{1}^{2}\left(K_{D 1}+\Gamma_{0} \Gamma_{1} K_{e}\right) & \Lambda_{1} \Gamma_{0} \Gamma_{1} K_{e} \\
\Lambda_{1} \Gamma_{0} \Gamma_{1} K_{e} & K_{D 1}+\Gamma_{0} \Gamma_{1} K_{e} \\
-\frac{1}{2} \Lambda_{1} K & -\frac{1}{2} K
\end{array}\right] \\
& N_{2_{12}}=\left[\begin{array}{ccc}
-\frac{1}{2} \Lambda_{1} K & 0 & -\frac{1}{2} \Lambda_{1} \Gamma_{0} \Gamma_{1} \\
-\frac{1}{2} K & 0 & -\frac{1}{2} \Gamma_{0} \Gamma_{1} \\
\Lambda_{2}^{2} K_{D 2} & 0 & 0
\end{array}\right] \\
& N_{2_{21}}=\left[\begin{array}{cc}
0 & 0 \\
-\frac{1}{2} \Lambda_{1} \Gamma_{0} \Gamma_{1} & -\frac{1}{2} \Gamma_{0} \Gamma_{1}
\end{array}\right] \\
& N_{2_{22}}=\left[\begin{array}{ccc}
0 & K_{D 2} & 0 \\
0 & 0 & \Gamma_{0} \hat{\Psi}^{\#}\left(\dot{q}_{1}\right) K_{e}^{-1}
\end{array}\right] \\
& D=\left[\begin{array}{c}
\Lambda_{1} \Gamma_{0} \Gamma_{1} \Delta_{\Psi}\left(\dot{q}_{1}\right) z \\
\Gamma_{0} \Gamma_{1} \Delta_{\Psi}\left(\dot{q}_{1}\right) z \\
0 \\
0 \\
-\Gamma_{0} K_{e}^{-1} \Delta_{\Psi}\left(\dot{q}_{1}\right) z
\end{array}\right]
\end{aligned}
$$

\footnotetext{
${ }^{1}$ Recalling that the matrices $\Gamma_{0}, \Gamma_{1}, K, \hat{\Psi}\left(\dot{q}_{1}\right), \Delta_{\Psi}\left(\dot{q}_{1}\right), K_{e}, \Lambda_{1}, \Lambda_{2}$, $K_{D 1}$ and $K_{D 2}$ are diagonal.
} 
where the matrix $\hat{\Psi}^{\sharp}\left(\dot{q}_{1}\right)$ is defined as:

$$
\hat{\Psi}^{\sharp}\left(\dot{q}_{1}\right)= \begin{cases}\frac{1}{\xi} & \dot{q_{1}} \in(-\varepsilon, \varepsilon) \\ \hat{\Psi}^{-1}\left(\dot{q}_{1}\right) & \text { other case }\end{cases}
$$

In fact matrix $\hat{\Psi}^{\sharp}\left(\dot{q}_{1}\right)$ is the inverse of $\hat{\Psi}\left(\dot{q}_{1}\right)$ except for $\dot{q}_{1}=$ 0 where the inverse in not defined. Equation (30) with a predefined tolerance $\varepsilon>0$ is used to avoid numerical difficulties when $\dot{q}_{1}$ tends to zero and consequently $\lambda_{\max }\left(N_{2}\right)$ tends to $\infty$.

The gains $K_{e}, K_{D 1}, K_{D 2}, \Lambda_{1}$ and $\Lambda_{2}$ may be chosen to make $N_{2}$ positive definite. A possible way of achieving this is to choose $K_{e}, K_{D 1}, K_{D 2}, \Lambda_{1}$ and $\Lambda_{2}$ such that $N_{2}$ becomes diagonal dominant.

Using the Rayleigh-Ritz theorem ${ }^{2}$ and the concept of uniform ultimate boundedness (Corless and Leitmann, 1991), it follows that

$$
\dot{V} \leq-\lambda_{\min }\left(N_{2}\right)\|\rho\|^{2}+\|\rho\|\|D\|
$$

According to (Canudas De Wit et al., 1995), due to the fact that $0<g\left(\dot{q}_{1}\right) \leq a$, if $\|z(0)\| \leq a$ then $\|z(t)\| \leq a, \forall t \geq 0$. In fact there exists an invariant set $\Omega=\{z:\|z\| \leq a\}$ so that all solutions of $z(t)$ starting in $\Omega$ remains in this set.

On the other hand, the approximation error: $\Delta_{\Psi}\left(\dot{q}_{1}\right)$ is a limited function. Due to these facts it is possible to define a upper bound $\bar{D}$ such that $\|D\| \leq \bar{D}$, then a condition for having $\dot{V}<0$ is

$$
\|\rho\|>\frac{\bar{D}}{\lambda_{\min }\left(N_{2}\right)}
$$

Since $\dot{V}<0$, the norm $\|\rho\|$ decreases until

$$
\|\rho\| \leq \frac{\bar{D}}{\lambda_{\min }\left(N_{2}\right)}
$$

This causes $\dot{V} \geq 0$ and forces the norm $\|\rho\|$ to increase. Eventually, the condition (32) is reached and $\dot{V}<0$ again.

The conclusion is that $V(t)$ is a decreasing function that converges to a fixed limit, implying that $V(t)$ is bounded and $\tilde{q}_{1}, \dot{\tilde{q}}_{1}, \tilde{q}_{2}, \dot{\tilde{q}}_{2}$, and $\dot{\tilde{z}}$ are bounded. The tracking errors converge to a residual set which depends on the friction coefficients and the controller gains.

\section{OFF-LINE IDENTIFICATION OF THE FRICTION PARAMETERS}

For the LuGre model, 6 parameters must be estimated for each link of the robot, that is, for the link $i$, the parameters $\Gamma_{0 i}, \Gamma_{1 i}, \Gamma_{2 i}, F_{c i}, F_{s i}, v_{s i}$ must be identified.

\footnotetext{
${ }^{2}$ For an hermitian matrix $A, x^{\mathrm{T}} A x \geq \lambda_{\min }(A) x^{\mathrm{T}} x$.
}

The estimation is done for each link at a time. All other joints are blocked and only the joint $i$ moves. The estimation proposed in (Canudas De Wit and Lischinsky, 1997) is done in two steps: the estimation of the static parameters $\Gamma_{2 i}, F_{c i}$, $F_{s i}$ and $v_{s i}$ and of the dynamic parameters $\Gamma_{0 i}$ and $\Gamma_{1 i}$.

\subsection{First Step: Static Parameters}

The static parameters of the LuGre model may be estimated using a static map. This static map ${ }^{3}$ is obtained from a series of experiments, where in each experiment the robot joint moves with a constant velocity and the friction torque is measured indirectly through the rotor current. Then, the static parameters are adjusted to the experimental data using nonlinear techniques.

One of these techniques is to solve a nonlinear least squares problem. In this case, supposing that $m$ experiments are made and that for each velocity $v_{j}$ a friction torque $F_{s s}\left(v_{j}\right)$ is measured (for $j=1, \ldots, m$ ), the objective is to find the parameters that minimize a cost function, that is, to solve

$$
\min _{F_{c i}, F_{s i}, v_{s i}, \Gamma_{2 i}}\left(\sum_{j=1}^{m}\left(F_{s s}\left(v_{j}\right)-\hat{F}_{s s}\left(v_{j}\right)\right)^{2}\right)
$$

where $\hat{F}_{s s}\left(v_{j}\right)$ are the estimated values for the friction torque, calculated according to the static friction model

$$
\hat{F}_{s s}\left(v_{j}\right)=\operatorname{sign}\left(v_{j}\right)\left(F_{c i}+\left(F_{s i}-F_{c i}\right) e^{-\left(\frac{v_{j}}{v_{s i}}\right)^{2}}\right)+\Gamma_{2 i} v_{j}
$$

\subsection{Second Step: Dynamic Parameters}

In order to determine the dynamic parameters of the friction model, it is important to perform an experiment in which the effects of these parameters stand out. In particular, the stickslip motion and the transient motions due to velocity reversals depend highly on $\Gamma_{0 i}$ and $\Gamma_{1 i}$. Therefore, these 2 parameters may be obtained from open-loop experiments that induce stick-slip motion and with velocity reversals. The dynamic parameters are adjusted to the experimental data using a nonlinear optimization method.

Supposing that an experiment is made and that $p$ samples are collected, that is, for each instant $t(k)$ a link angular position $x(t(k))$ is measured (for $k=1, \ldots, p$ ), the objective is to find the parameters that minimize a cost function in the form

$$
\min _{\Gamma_{0 i}, \Gamma_{1 i}}\left(\sum_{k=1}^{p}(x(t(k))-\hat{x}(t(k)))^{2}\right)
$$

\footnotetext{
${ }^{3}$ An example of a static map is given in Fig. 6.
} 
where $\hat{x}(t(k))$ is the estimated value of the link angular position obtained by numerical integration from the model

$$
\begin{gathered}
J_{x} \ddot{\hat{x}}=u-\hat{F}_{i} \\
\dot{\hat{z}}_{i}=\dot{\hat{x}}-\Gamma_{0 i} \frac{|\dot{\hat{x}}|}{g(\dot{\hat{x}})} \hat{z}_{i} \\
\hat{F}_{i}=\Gamma_{0 i} \hat{z}+\Gamma_{1 i} \dot{\hat{z}}+\Gamma_{2 i} \dot{\hat{x}}
\end{gathered}
$$

where $J_{x}$ represents the sum of the inertia in the rotor and in the link and $u$ is the torque applied to the link (the values of the static parameters $\Gamma_{2 i}, F_{c i}, F_{s i}$ and $v_{s i}$ identified in the first step are used here). It is important to notice that, since the robot is approximated by a rigid model (37), the experiment must be performed in low velocities, in order to not excite the flexible transmission dynamics.

\section{EXPERIMENT: CASCADE CONTROL OF A FLEXIBLE JOINT ROBOT}

In this section, it is described the experiment performed, which consists in the implementation of the cascade control with friction compensation proposed in Section (3) to a planar flexible joint robot with one link and one transmission. A drawing of the robot considered in this work is shown in Figure 3. Although it has two links, here only the first link is studied.

\subsection{Hardware Description}

The hardware set-up is shown in Figure 4.

In this application, the $\operatorname{SPACE} \mathrm{DS} 1102$ system is used to control the manipulator. The controller is implemented in a personal computer using the Simulink/MATLAB interface. The controller reads the rotor angular positions in real-time from two Hewlett Packard HEDS-6010 high resolution incremental encoders. Comparing the actual position and the

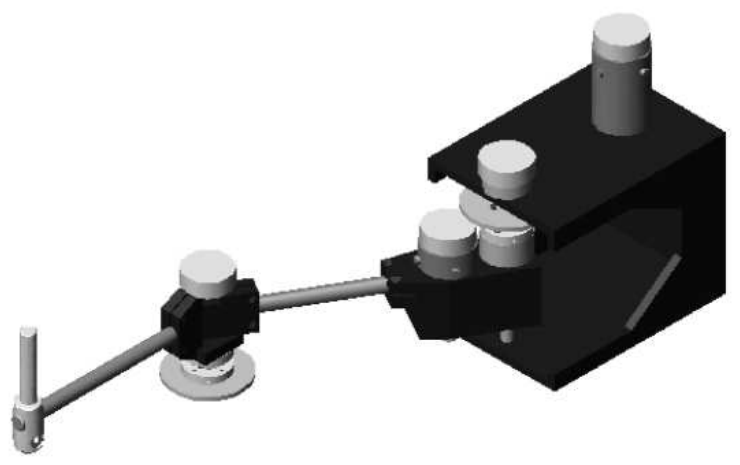

Figure 3: Planar flexible joint robot.

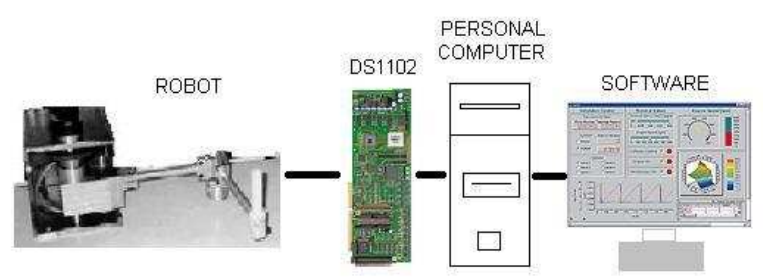

Figure 4: Hardware set-up.

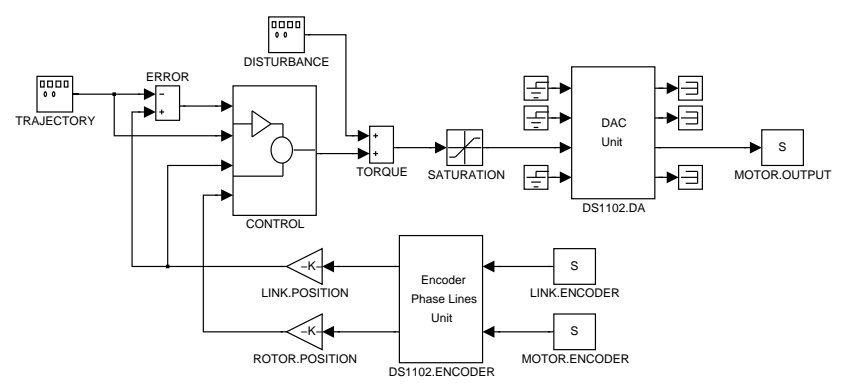

Figure 5: Simulink implementation of the controller blocks.

reference, the controller calculates the control torque that must be sent back to the robot using the 12 bits Digital to Analog Converter outputs. The 12A PWM Advanced Motion Controls servo amplifier drives the necessary current to the motor. In this application the servo amplifier is used in torque mode (voltage input to current output). Figure 5 shows the structure of the controller.

The control block represents the cascade control algorithm. Link.position and Rotor.position blocks represent the link and rotor actual positions, respectively. The Trajectory block generates the desired trajectory. The desired link position $q_{1 d}$ and its derivatives, up to $3^{\text {rd }}$ order, are uniformly bounded. The DAC unit and the Encoder Phase Lines unit are $d S P A C E$ drivers that perform the digital to analog conversion and the digital interpretation of the encoder values, respectively.

\subsection{Model of the Planar Flexible Joint Robot with One Link}

Using the simplified model (8), the planar robot with one link and one flexible transmission can be represented by

$$
\begin{aligned}
& I \ddot{q}_{1}+F=K\left(q_{2}-q_{1}\right) \\
& J \ddot{q}_{2}+K\left(q_{2}-q_{1}\right)=\tau
\end{aligned}
$$

where $I$ is the inertia of the link and the load (a constant value), $q_{1}$ is the joint position, $q_{2}$ is the rotor position, $J$ is 
the rotor inertia, $K$ is the spring constant, $F$ is the friction torque and $\tau$ is the control torque. The inertia and spring constant were obtained using indirect measurements. The spring constant, in particular, was obtained using a suitable experiment (Seto, 1964).

The nominal parameters for the first link of the manipulator are given in Table 1.

Table 1: First Link Parameters:

\begin{tabular}{|c|c|}
\hline Parameter & Value \\
\hline$I:$ Link Inertia $\left(\mathrm{kg} \mathrm{m}^{2}\right)$ & 0.07 \\
\hline$J:$ Rotor Inertia $\left(\mathrm{kg} \mathrm{m}^{2}\right)$ & 0.005 \\
\hline$K:$ Spring Constant $(\mathrm{Nm} / \mathrm{rad})$ & 6.77 \\
\hline
\end{tabular}

\subsection{Identification of Friction Parameters}

The identification of the friction parameters was done according to the procedure described in Section 5. The encoder resolution was 0.72 degrees. The velocity resolution for measuring static values was $0.1 \mathrm{rad} / \mathrm{s}$. The static map obtained for the first link of the robot manipulator is shown in Fig. 6.

Using the Matlab function lsqcurvefit to solve the optimization problem (34), the static parameters are estimated as $F_{s 1}=0.15 \mathrm{Nm}, F_{c 1}=0.11 \mathrm{Nm}, v_{s 1}=0.05 \mathrm{rad} / \mathrm{s}$ and $\Gamma_{21}=0.2 \mathrm{Nms} / \mathrm{rad}$. Fig. 6 shows a non symmetric friction with velocity, but in this paper symmetric values were used, like in (Canudas De Wit and Lischinsky, 1997).

Fig. 7 and Fig. 8 illustrate the open-loop experiment performed for the determination of the dynamic parameters.

Fig. 7 shows the torque input and Fig. 8 the measured angular position. By solving the problem optimization problem 36, the dynamic parameters are estimated as $\Gamma_{01}=100$

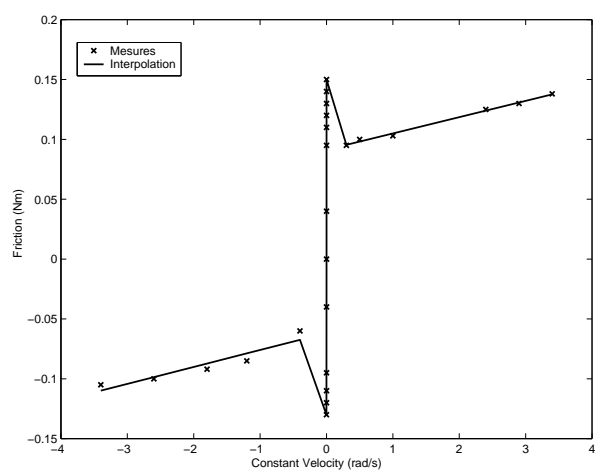

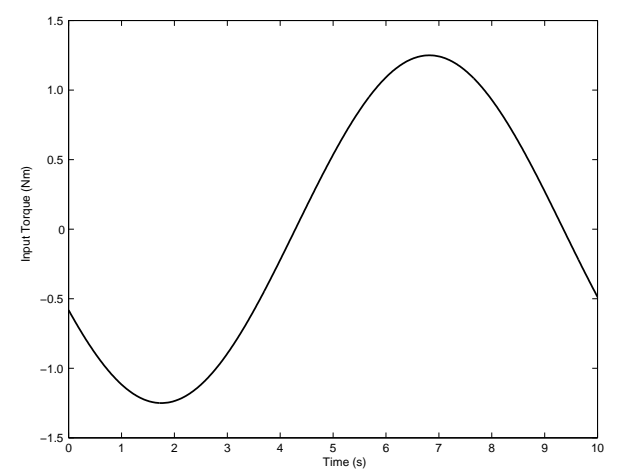

Figure 7: Open-loop experiment: input torque.

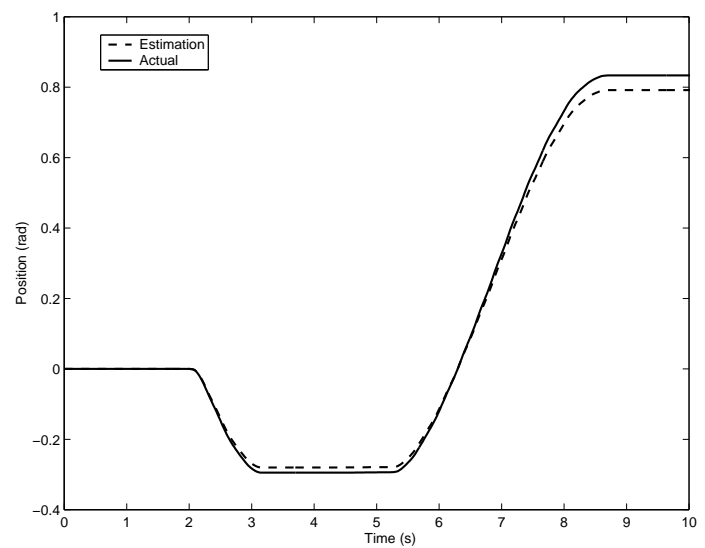

Figure 8: Open-loop experiment: measured position and estimated position.

$\mathrm{Nm} / \mathrm{rad}$ and $\Gamma_{11}=0.1 \mathrm{Nms} / \mathrm{rad}$. A simulation of the link angular position using these estimated parameters is also shown in Fig. 8.

The estimated values of the 6 parameters of the LuGre model for the first link are given in Table 2.

Table 2: Estimated Parameters of the LuGre Model:

\begin{tabular}{|c|ll|}
\hline Parameter & \multicolumn{2}{|c|}{ Value } \\
\hline$F_{s 1}$ & $0.15 \mathrm{Nm}$ \\
$F_{c 1}$ & $0.11 \mathrm{Nm}$ \\
$v_{s 1}$ & $0.05 \mathrm{rad} / \mathrm{s}$ \\
$\Gamma_{11}$ & $100 \mathrm{Nms} / \mathrm{rad}$ \\
$\Gamma_{01}$ & $0.1 \mathrm{Nm} / \mathrm{rad}$ \\
$\Gamma_{21}$ & 0.2 & $\mathrm{Nms} / \mathrm{rad}$ \\
\hline
\end{tabular}

Figure 6: Static map of velocity versus friction torque. 


\subsection{The Cascade Control Strategy}

In this experiment, it is used the cascade control proposed in Section 3, adapted to the model (40) of the planar flexible joint robot considered here.

From (13) (18) and (19), the control $u_{d}$ is given by

$$
u_{d}=I \ddot{q}_{r 1}-K_{D 1} s_{1}+\hat{F}
$$

and, from (16), the control torque $\tau$ is

$$
\tau=J\left(\ddot{q}_{2 d}-\Lambda_{2} \dot{\tilde{q}}_{2}\right)-K_{D 2} s_{2}+K\left(q_{2}-q_{1}\right)
$$

Equations (41) and (42) use only accelerations and jerks of the reference signal. The control gains were chosen to obtain a response without actuator vibrations and with a sufficiently smooth control signal. Table 3 shows the controller gains.

Table 3: Controller Gains:

\begin{tabular}{|c|c|c|c|c|c|c|}
\hline Gain & $K_{e}$ & $K_{D 1}$ & $K_{D 2}$ & $k_{v 1}$ & $\Lambda_{1}$ & $\Lambda_{2}$ \\
\hline Value & 0.001 & 1.0 & 1.0 & 2.0 & 200.0 & 6.0 \\
\hline
\end{tabular}

\subsection{Experimental Results}

The tests were performed using two different trajectories. The link and rotor control were given by equations (41) and (42), respectively. The velocities were obtained using a filter and a numeric derivative process.

Figure 9 shows the system response using the cascade controller without friction compensation.

In order to outline the friction effect, Figure 10 shows the improved system response when using friction compensation.

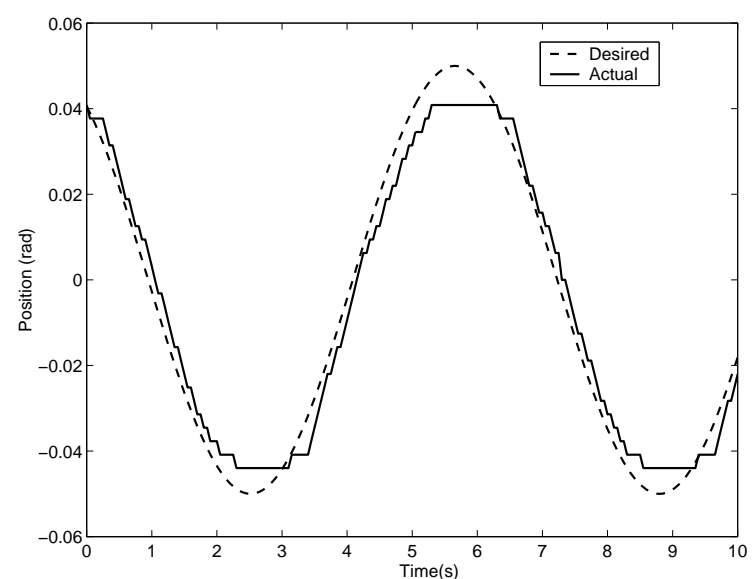

Figure 9: Cascade control without friction compensation.

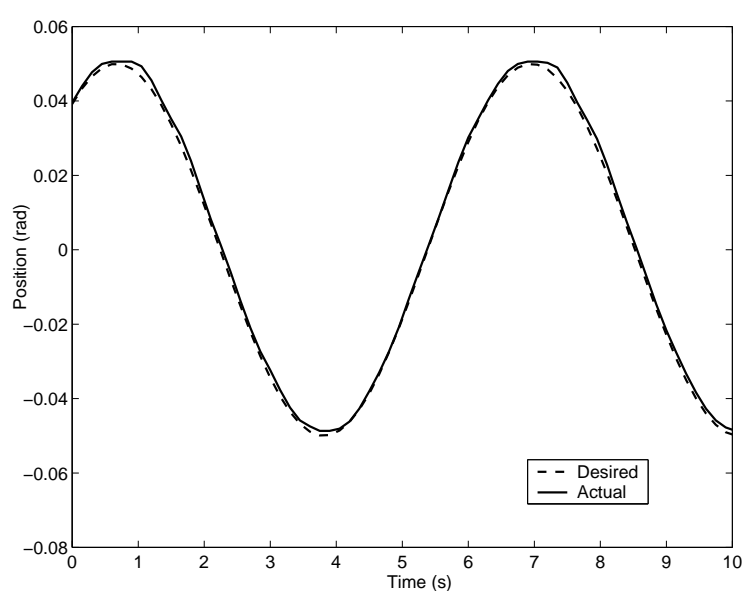

Figure 10: Cascade control with friction compensation.

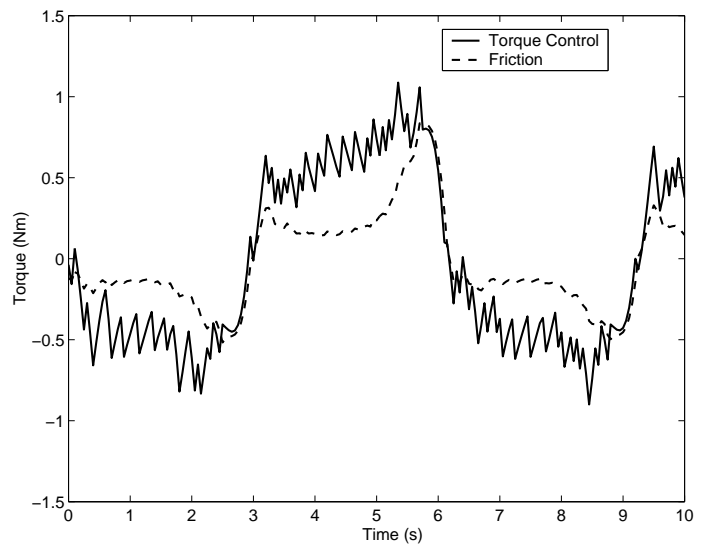

Figure 11: Torque control and the estimated friction torque.

Figure 11 shows that the control torque strongly depends on the estimated friction torque.

Figure 12 shows the actual and desired link positions using a polynomial trajectory without friction compensation.

Figure 13 shows the improved system response when using the cascade controller with friction compensation.

The tracking errors for both cases are shown in Figure 14.

Figure 15 shows a faster dynamic motion experiment, which allows to better assess the performance of the cascade control algorithm with respect to the joint flexibilities. It is possible to see that the results remain good.

Obviously, since friction varies with temperature, load and wear, besides the direction of motion, friction compensation results cannot be assured in other tests, without refining parameter identification. 


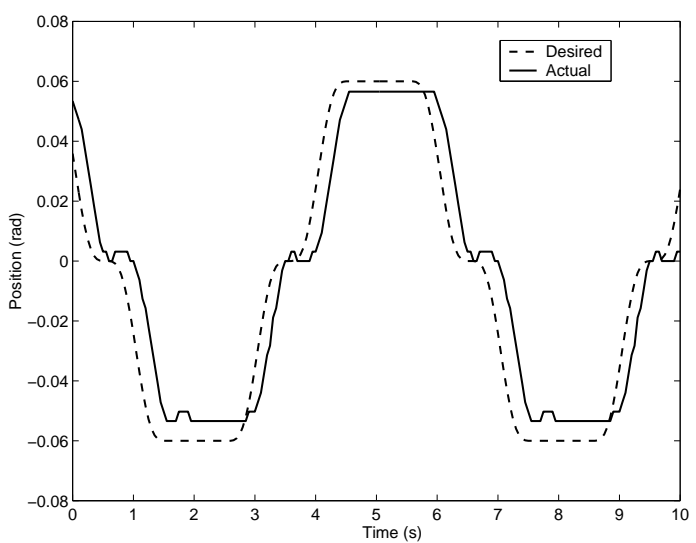

Figure 12: Cascade control without friction compensation.

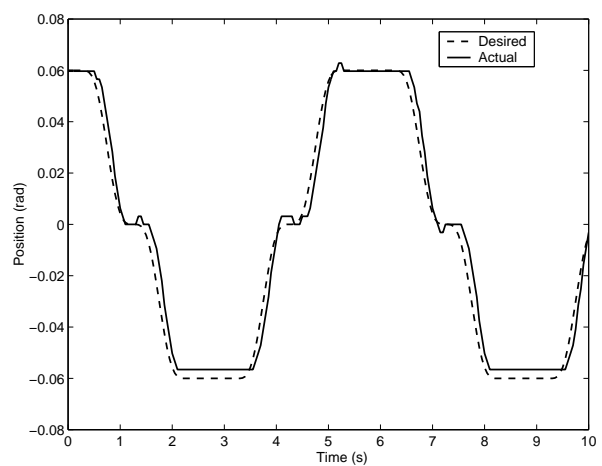

Figure 13: Cascade control with friction compensation.

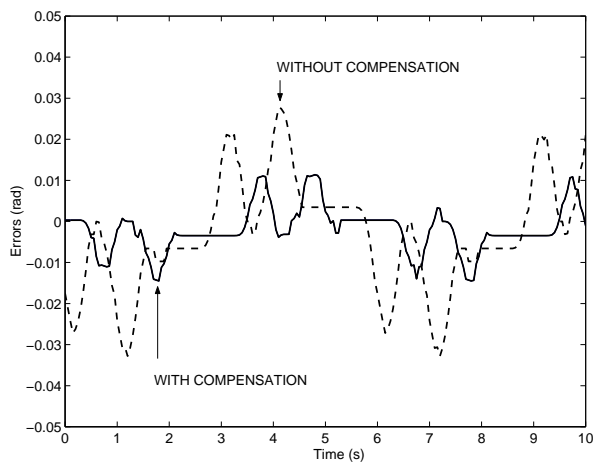

Figure 14: Tracking errors.

\section{CONCLUDING REMARKS}

In this paper, a systematic procedure to identify the friction model parameters and to experimentally analyse the position tracking control strategy performance, when implemented to a flexible joint robot prototype, has been presented. The proposed cascade control methodology uses the LuGre friction model without any assumption about the actuator's torque

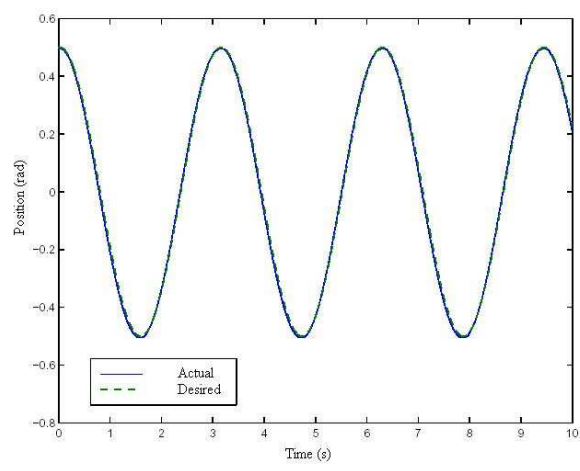

Figure 15: Cascade control compensation.

response. The convergence of the tracking errors was theoretically proved as well as the experimental results demonstrate the effectiveness of the friction compensation. Further works will consider adaptive procedures to update the parameters of the friction observer, the problem of design control gains to meet performance, the influence of perturbations and the study of the force control problem in robots with elastic joints.

\section{APPENDIX A. SYMBOL NOTATION AND MODEL PARAMETERS}

In this appendix the simplified model of a flexible joint robot with friction terms and a friction force model are revisited and the parameters described in table 4 and table 5. The flexible robot model with the friction parameters is given by:

$$
\begin{aligned}
M\left(q_{1}\right) \ddot{q}_{1}+C\left(q_{1}, \dot{q}_{1}\right) \dot{q}_{1}+G\left(q_{1}\right)+F & =K\left(q_{2}-q_{1}\right) \\
J \ddot{q}_{2}+K\left(q_{2}-q_{1}\right) & =\tau
\end{aligned}
$$

The friction force model is given by:

$$
F=\Gamma_{0} z+\Gamma_{1} \dot{z}+\Gamma_{2} \dot{q}_{1}
$$


Table 4: Flexible Robot Model with Friction Parameters

\begin{tabular}{ll}
\hline Symbol & Name \\
\hline$M\left(q_{1}\right)$ & Inertia Matrix \\
$J=\operatorname{diag}\left(J_{1}, \cdots, J_{n}\right)$ & Rotor inertia matrix \\
$C\left(q_{1}, \dot{q}_{1}\right)$ & Centrifuge and coriolis \\
& terms \\
$G\left(q_{1}\right)$ & Gravitational torques \\
$K=\operatorname{diag}\left(K_{1}, \cdots, K_{n}\right)$ & Spring matrix coefficients \\
$\tau$ & Control inputs \\
$q_{1}, \dot{q}_{1}, \ddot{q}_{1}$ & Link position, velocity and \\
$q_{2}, \dot{q}_{2}, \ddot{q}_{2}$ & acceleration \\
$F$ & Rotor position, velocity and \\
$F$ & acceleration \\
\hline
\end{tabular}

Table 5: Friction Model Parameters

\begin{tabular}{|c|c|}
\hline Symbol & Name \\
\hline$z$ & $\begin{array}{l}\text { An internal state that describes the average } \\
\text { elastic deflection of the contact surfaces dur- } \\
\text { ing the stiction phases }\end{array}$ \\
\hline$\dot{z}$ & $\begin{array}{l}\text { An internal state that describes the average } \\
\text { elastic deflection velocity of the contact sur- } \\
\text { faces during the stiction phases }\end{array}$ \\
\hline$\Gamma_{0}$ & $\begin{array}{l}\text { A diagonal matrix of the stiffness coefficients } \\
\text { of the microscopic deformation } z \text { during the } \\
\text { pre-sliding displacements }\end{array}$ \\
\hline$\Gamma_{1}$ & $\begin{array}{l}\text { A diagonal matrix of the damping coefficients } \\
\text { associated to } \dot{z}\end{array}$ \\
\hline$\Gamma_{2}$ & $\begin{array}{l}\text { A diagonal matrix representing the viscous } \\
\text { friction }\end{array}$ \\
\hline
\end{tabular}

\section{REFERENCES}

Al-Bender, F., Lampaert, V. and Swevers, J. (2005). The generalized Maxwell-slip model: a novel model for friction simulation and compensation, IEEE Transactions on Automatic Control 50(11): 1883-1887.

Albu-Schäffer, A. and Hirzinger, G. (2001). Parameter identification and passivity based joint control for a 7 dof torque controlled light weight robot, In proc. International Conference on Robotics and Automation, Seoul, Korea pp. 1087-1093.

Armstrong-Hélouvry, B. (1993). Stick-slip and control in low-speed motion, IEEE Transactions on Automatic Control 38(10): 1483-1496.
Brogliato, B., Ortega, R. and Lozano, R. (1995). Global tracking controllers for flexible-joint manipulators: a comparative study, Automatica 31(7): 941-956.

C. Canudas de Wit, R. K. (2007). Passivity analysis of a motion control for robot manipulators with dynamic friction, Asian Journal of Control 9(1): 30-36.

Canudas De Wit, C. and Lischinsky, P. (1997). Adaptive friction compensation with partially known dynamic model, International Journal of Adaptive Control and Signal Processing 11: 65-80.

Canudas De Wit, C., Olsson, H., Åström, K. J. and Lischinsky, P. (1995). A new model for control systems with friction, IEEE Transactions on Automatic Control 40(3): 419-425.

Canudas De Wit, C., Siciliano, B. and Bastin, G. (1996). Theory of Robot Control, Springer - Verlag London Limited.

Corless, M. and Leitmann, G. (1991). Continuous state feedback guaranteeing uniform ultimate boundedness for uncertain dynamic systems, IEEE Transactions on Automatic Control 26(5): 1139-1144.

Dupont, P. E. (1994). Avoiding stick-slip through PD control, IEEE Transactions on Automatic Control 39(5): 10941097.

Fantoni, I. and Lozano, R. (2000). Adaptive stabilization of underactuated flexible-joint robots using an energy approach and min-max algorithms, In Proceedings of the American Control Conference pp. 2511-2512.

Gandhi, P. S., Ghorbel, F. H. and Dabney, J. (2002). Modeling, identification, and compensation of friction in harmonic drives, Proc. of the 41st IEEE Conf. on Decision and Control .

Ghorbel, F. and Spong, M. W. (2000). Integral manifolds of singularly perturbed systems with application to rigid-link flexible-joint multibody systems, International Journal of Nonlinear Mechanics June(34): 133155.

Gomes, S. C. P. and Santos da Rosa, V. (2003). A new approach to compensate friction in robotic actuators, Robotics and Automation, 2003. Proceedings. ICRA'03. IEEE International Conference on 1: 622627.

Guenther, R. and Hsu, L. (1993). Variable struture adaptative cascade control of rigid-link electrically driven robot manipulators, Proc. of IEEE Conf. on Decision and Control . 
Guenther, R. and Perondi, E. (2002). The pneumatic positioning system cascade control with friction compensation, In Proceedings of the Congresso Brasileiro de Automatica pp. 100-106.

Guenther, R. and Perondi, E. (2004). O controle em cascata de sistemas pneumáticos de posicionamento, $R e$ vista SBA Controle e Automação 15(2): 149-161.

Guenther, R., Perondi, E., De Pieri, E. R. and Valdiero, A. C. (2006). Cascade controlled pneumatic positioning system with lugre model based friction compensation, Journal of the Brazilian Society of Mechanical Sciences and Engineering, 28(1): 48-57.

Huang, L., Ge, S. S. and Lee, T. H. (2006). Position/force control of uncertain constrained flexible joint robots, Mechatronics 16(2): 111-120.

Jeon, S. and Tomizuka, M. (2005). Limit cycles due to friction forces in flexible joint mechanisms, Advanced Intelligent Mechatronics. Proceedings, 2005 IEEE/ASME International Conference on pp. 723-728.

Kokotovic, P. and Sussmann, P. (1989). A positive real condition for global stabilization of nonlinear systems, Systems and Control Letters 13(2): 125-133.

Lischinsky, P., Canudas-De-Wit, C. and Morel, G. (1999). Friction compensation for an industrial hydraulic robot, IEEE Control Systems Feb.: 25-32.

Lozano, R., Valera, A., Albertos, P. and Arimoto, S. (1997). PD control of robot manipulators considering joint flexibility actuators dynamic and friction, In Proceedings of the American Control Conference pp. 2638-2641.

Nicosia, S. and Tomei, P. (1988). On the feedback linearization of robots with elastics joints, In Proceedings of the IEEE International Conference on Robotics and Automation pp. 180-185.

Nicosia, S. and Tomei, P. (1992). A method to design adaptive controllers for flexible joint robots, In Proc. IEEE Int. Conf. on Robotics and Automation pp. 701-706.

Ramirez, A. R. G., De Pieri, E. R. and Guenther, R. (2002). Experimental study applied to an industrial robot by using variable structure controllers and friction compensation, Journal of the Brasilian Society of Mechanical Sciences XXIV(4): 302-309.

Ramirez, A. R. G., De Pieri, E. R. and Guenther, R. (2003). Controle em cascata de um manipulador robótico com um elo e uma transmissão flexível, Revista SBA Controle e Automação 14(4): 393-401.

Readman, M. C. (1994). Flexible Joint Robots, CRC Press.
Seto, W. (1964). Theory and Problems of Mechanical Vibrations, McGraw Hill.

Spong, M. W. (1987). Modelling and control of elastic joint robots, ASME, Transactions: Journal of Dynamic Systems, Measurement, and Control 109: 310-319.

Spong, M. W. and Vidyasagar, M. (1989). Robot Dynamics and Control, Prentice Hall, Inc., New Jersey.

Tomei, P. (1991). A simple PD controller for robots with elastic joints, IEEE Transactions on Automatic Control 36(10): 1208-1213.

Tomei, P. (2000). Robust adaptive friction for tracking control of robot manipulators, IEEE Transactions on Automatic Control 45(11): 2164-2169.

Wang, Z. and Khorrami, F. (2000). Robust trajectory tracking for manipulators with joint flexibility via backstepping, In Proceedings of the American Control Conference pp. 2849-2853.

Xie, W. F. (2007). Sliding-mode-observer-based adaptive control for servo actuator with friction, IEEE Transactions on Industrial Electronics 54(3): 1517-1527. 